Journal of Patient-Centered

$11-3-2014$

\title{
Which Clinical Guidelines Should Be Implemented for Management of Osteopenia in Primary Care? A Clin-IQ
}

Turia Hollingsworth

Follow this and additional works at: https://aah.org/jpcrr

Part of the Geriatrics Commons, Musculoskeletal Diseases Commons, and the Osteopathic Medicine and Osteopathy Commons

\section{Recommended Citation}

Hollingsworth T. Which clinical guidelines should be implemented for management of osteopenia in primary care? A Clin-IQ. J Patient Cent Res Rev. 2014;1:201-203. doi: 10.17294/2330-0698.1041

Published quarterly by Midwest-based health system Advocate Aurora Health and indexed in PubMed Central, the Journal of Patient-Centered Research and Reviews (JPCRR) is an open access, peer-reviewed medical journal focused on disseminating scholarly works devoted to improving patient-centered care practices, health outcomes, and the patient experience. 


\section{Which Clinical Guidelines Should Be Implemented for Management of Osteopenia in Primary Care? A Clin-IQ}

Turia Hollingsworth, MD

Department of Family Medicine, Aurora St. Luke's Medical Center, Aurora Health Care, Milwaukee, WI

\begin{abstract}
Literature review was conducted to determine clinical guidelines for osteopenia management of immunocompetent patients in the primary care setting. It was concluded that pharmacological treatment should be offered to patients with osteopenia if they are considered high risk, which can be determined by utilizing the World Health Organization Fracture Risk Assessment Tool $\left(\right.$ FRAX $\left.^{\circledR}\right)$ to evaluate 10year risk. Patients with a calculated hip fracture probability of at least $3 \%$ or those with probability of $20 \%$ or more for all osteoporotic fractures may be offered bisphosphonate therapy. (J Patient-Centered Res Rev. 2014;1:201-203.)
\end{abstract}

\section{Keywords}

osteopenia, treatment, guidelines, osteoporosis

\section{Clinical Question}

Which clinical guidelines for management of osteopenia should be implemented in primary care?

\section{Answer}

Pharmacological treatment with bisphosphonates should be offered to patients with osteopenia who are considered high risk for osteoporotic fractures. This can be determined by identifying patients with prevalent fractures or by utilizing the World Health Organization Fracture Risk Assessment Tool (FRAX ${ }^{\circledR}$ ) in patients with no present fracture to determine their 10-year risk. Osteopenic patients not considered high risk should be advised on lifestyle modifications as well as vitamin $\mathrm{D}$, calcium and other mineral supplementation if clinically indicated.

Date answer was determined: March 2014

Level of evidence: $\mathrm{IIb}$

\section{Inclusion Criteria}

MEDLINE, year of publication from 2000 to 2014, observational, cohort

Correspondence: Turia Hollingsworth, MD, 136 N. Milwaukee Street, \#102, Milwaukee, WI, 53233, Phone: 414-294-8351, Email: turia.hollingsworth@aurora.org

\section{Exclusion Criteria}

Human immunodeficiency virus (HIV), chronic obstructive pulmonary disease, female triad (female athletes with low energy availability, amenorrhea and decreased bone mineral density), patients on chronic steroids, rheumatoid arthritis

\section{Summary of Problem}

Osteoporotic fractures can be devastating for individuals, and they also represent a significant economic burden in health care. There were an estimated 9 million osteoporotic fractures in 2000. ${ }^{1}$ Hip fractures increase the risk of mortality and morbidity in the elderly, and approximately $50 \%$ of patients are unable to return to independent living. ${ }^{2}$

Based on World Health Organization (WHO) diagnostic classification, osteoporosis is diagnosed by a bone mineral density (BMD) that is 2.5 or more standard deviations (SD) below the BMD of a young adult reference population. Osteopenia is diagnosed by BMD of 1-2.49 SD below the young adult reference. Patients in the osteoporotic range are at a significantly increased risk for future fracture and therefore require therapy. However, patients with osteopenia are also at increased risk for future fracture when compared to the general population. The National Osteoporosis Risk Assessment (NORA) study, which analyzed 200,160 women, showed that postmenopausal women with BMD $\leq-2.5 \mathrm{SD}$ had a 2.74 times higher 1-year risk of fracture and that women with osteopenia had a 1.73 times higher risk of fracture compared to women with normal BMD. ${ }^{3}$

Although it is apparent that patients with osteopenia are at higher risk of fracture than the general population, determining which patients in this group should be targeted for additional management has been perplexing for clinicians. WHO has acknowledged that BMD should not be the sole determining factor in treatment, and in 2008 it developed the Fracture Risk Assessment Tool (FRAX ${ }^{\circledR}$ ) to determine absolute fracture risk in an effort to assist providers in treatment decisions. ${ }^{4}$

The FRAX tool (http://www.shef.ac.uk/FRAX) provides individual patient models developed from population-based 
cohorts to assess future fracture risk in both men and women. It incorporates clinical risk factors with or without BMD to calculate 10-year risk of fracture. The National Osteoporosis Foundation (NOF) also has provided guidance on how to manage patients with osteopenia, and it now supports using FRAX to determine whether treatment is indicated in this group. ${ }^{5}$

\section{Summary of Evidence}

Several studies have shown that fractures occur even before patients reach the osteoporotic range. Siris et al. used data from the NORA study to analyze the development of fractures over 1 year in 149,524 white postmenopausal women (age 50-104 years, mean age 64.5). ${ }^{6}$ They found that $82 \%$ of reported new fractures were in women with T-scores greater than -2.5 (i.e. nonosteoporotic as defined by WHO) and $67 \%$ with T-scores greater than -2.0 . Only $6.4 \%$ of women had T-scores of -2.5 or less, and although fracture rates were higher in this group, these women accounted for only $18 \%$ of the osteoporotic fractures and $26 \%$ of hip fractures. If the NOF treatment guidelines are implemented (i.e. when T-score is $\leq-2$, or when T-score is $\leq-1.5$ plus one or more clinical risk factors per FRAX), 23\% of women met this criteria. In this group, fracture rates were lower but accounted for $45 \%$ of osteoporotic fractures and $53 \%$ of hip fractures. ${ }^{6}$ The results of this study highlight that if osteoporotic treatment is based solely on BMD $\leq-2.5$, several patients at risk may be missed.

It is clear that certain patients in the osteopenia range also may require pharmacological treatment. However, there is also the question of how cost-effective would it be to treat all patients with osteopenia. Schousboe et al. investigated the cost-effectiveness of 5-year therapy with alendronate in postmenopausal women (age 55-75 years) with T-scores ranging from -1.5 to -2.4 . They found that pharmacotherapy with alendronate was cost-effective in women age 55-75 with BMD from -2.0 to -2.4 , but was not cost-effective in women with BMD of -1.5 who had no history of clinical fractures and no risk factors for fracture. ${ }^{7}$

NOF recommends pharmacological therapy with bisphosphonates for patients with hip or vertebral fractures, either clinical or asymptomatic, as these patients are at increased risk for future fracture. NOF also endorses use of FRAX to determine absolute risk of fracture in patients with osteopenia and suggests that treatment is cost-effective in patients with 10 -year hip fracture probability of $\geq 3 \%$ or probability of $\geq 20 \%$ for all osteoporotic fractures. ${ }^{5}$ The American Congress of Obstetricians and Gynecologists also recently recommended similar guidelines, with implementation of FRAX for individuals with BMD between -1 and $-2.5 .{ }^{8}$ The 2011 U.S. Preventive Services Task Force guidelines for osteoporosis recommend screening for women 65 years or older or in younger women with an equal or greater fracture risk. They used FRAX to assess fracture risk and determined that for women at least 65 years of age with no risk factors, the 10 -year risk was 9.3\%. Therefore, the U.S. Preventive Services Task Force recommended using 9.3\% 10-year risk to screen women age 50-64 years. ${ }^{9}$ However, there are still no guidelines to determine management of women in the osteopenic range. Similarly, there are no current estimates of the number needed to treat for benefit (NNT) in those specifically with osteopenia. Using the findings from the NORA study ( $70 \%$ higher risk of fracture when compared to women with normal BMD), ${ }^{3}$ the calculations of Nelson et al. ${ }^{10}$ could be used. In this case, for women age 60-64 years, the NNT would be 72 to prevent one hip fracture. Similarly, while the number needed to harm has not been specifically calculated, the risks of treatment are not insignificant. ${ }^{9}$

Dawson-Hughes et al. ${ }^{11}$ evaluated the effect of incorporating FRAX into treatment guidelines. Results showed that patients age $\geq 65$ years with prior fracture alone had a high 10-year hip fracture probability of 3\% and therefore warranted treatment. Patients with BMD T-score $\leq-2.0$ with prior fracture also had 10-year risk meeting or exceeding $3 \%$ (those with $\mathrm{T}$ score $\leq-2.0$ had $2.7 \%$ 10-year risk, and those with T-score $\leq-2.5$ had $4.7 \% 10$-year risk). Patients with osteopenia (T-score of -2.0) and at least one risk factor in addition to prior fracture also meet criteria for treatment (fracture probability 2.9-5.0\%). ${ }^{11}$ These data demonstrate how FRAX is able to use risk stratification in conjunction with BMD to provide better identification and management guidelines for patients with osteopenia.

FRAX was revised in 2009 based on updated epidemiological data for the United States. This was performed in order to reflect decreasing hip fracture incidence among the country's white population. Results revealed that there was a strong correlation $(r>0.99)$ at all ages between the two versions for estimates of fracture probability. However, the revised model gives lower median probabilities. For major osteoporotic fracture probability, the median value was lower by $13-24 \%$ in men and by $19-24 \%$ in women. For hip fracture probability, the median value was lower by $40 \%$ and $27 \%$ in men and by $43 \%$ and $30 \%$ in women at age 50 and 60 years, respectively. At age 70, both the models gave similar probabilities for hip fracture. These lower probabilities reflect the decreased incidence in the population and have little impact on the ability to calculate risk of fracture. ${ }^{12}$ 


\section{Conclusions}

The guidelines endorsed by the National Osteoporosis Foundation and World Health Organization should be adopted in the primary care setting. When evaluating patients with osteopenia, bone mineral density along with clinical risk factors should be assessed using the FRAX tool. This allows management of osteopenia to be individualized based on 10 -year risk. Patients with hip fracture probability of $\geq 3 \%$ or probability of $\geq 20 \%$ for all osteoporotic fractures should be offered pharmacological treatment with bisphosphonates to prevent fractures and associated poor outcomes.

\section{Acknowledgments}

The Clin-IQ teaching materials used for this project were generously provided by Oklahoma Shared Clinical \& Translational Resources, funded by grant number NIGMS U54GM104938, NIGMS/NIH.

\section{Conflicts of Interest}

None.

\section{References}

1. Johnell O, Kanis JA. An estimate of the worldwide prevalence and disability associated with osteoporotic fractures. Osteoporos Int. 2006;17:1726-33.

2. Morrison RS, Chassin MR, Siu AL. The medical consultant's role in caring for patients with hip fracture. Ann Intern Med. 1998;128:1010-20.
3. Siris ES, Miller PD, Barrett Connor E, et al. Identification and fracture outcomes of undiagnosed low bone mineral density in postmenopausal women: results from the National Osteoporosis Risk Assessment. JAMA. 2001;286:2815-22.

4. World Health Organization. WHO Scientific Group on the Assessment of Osteoporosis at the Primary Health Care Level. www.who.int/chp/ topics/Osteoporosis.pdf. Accessed March 8, 2014.

5. National Osteoporosis Foundation. Clinicians Guide to Prevention and Treatment of Osteoporosis. Washington, DC: National Osteoporosis Foundation; 2014.

6. Siris ES, Chen YT, Abbott TA, et al. Bone mineral density thresholds for pharmacological intervention to prevent fractures. Arch Intern Med. 2004;164:1108-12.

7. Schousboe JT, Nyman JA, Kane RL, Ensrud KE. Cost-effectiveness of alendronate therapy for osteopenic postmenopausal women. Ann Intern Med. 2005;142:734-41.

8. Committee on Practice Bulletins-Gynecology, The American College of Obstetricians and Gynecologists. ACOG Practice Bulletin N. 129. Osteoporosis. Obstet Gynecol. 2012;120:718-34.

9. U.S. Preventive Services Task Force. Screening for osteoporosis: U.S. Preventive Services Task Force recommendation statement. Ann Intern Med. 2011;154:356-64.

10. Nelson HD, Helfand M, Woolf SH, Allan JD. Screening for postmenopausal osteoporosis: a review of the evidence for the U.S. Preventive Services Task Force. Ann Intern Med. 2002;137:529-41.

11. Dawson-Hughes B, Tosteson AN, Melton LJ 3rd, et al. Implications of absolute fracture risk assessment for osteoporosis practice guidelines in the USA. Osteoporos Int. 2008;19:449-58.

12. Kanis JA, Johansson H, Oden A, Dawson-Hughes B, Melton LJ 3rd, McCloskey EV. The effects of a FRAX revision for the USA. Osteoporos Int. 2010;21:35-40.

(C) 2014 Aurora Health Care, Inc. 